\title{
HIV RESISTANCE TO ANTIRETROVIRAL DRUGS: MECHANISMS, GENOTYPIC AND PHENOTYPIC RESISTANCE TESTING IN CLINICAL PRACTICE
}

\author{
P. Blaise, ${ }^{1}$ P. Clevenbergh, ${ }^{2}$ D. Vaira,${ }^{3}$ M. Moutschen,,${ }^{4}$ P. Dellamonica ${ }^{5}$
}

Keywords: HIV drug resistance. Genotypic resistance testing. Phenotypic resistance testing, Antiretrovirals.

\begin{abstract}
HIV resistance to antiretroviral agents is a major contributory cause of treatment failure. The dynamics of HIV replication, together with patient-, physician-, and drug-related factors, lead to emergence of HIV resistant strains in most of the patients.

Phenotypic assays look for an increase in the antiretroviral drug (ARV) concentration that inhibits $50 \%$ of the growth of the tested HIV strain $\left(\mathrm{IC}_{50}\right)$, comparatively with a reference strain cultivated in parallel.

Genotypic tests detect resistance mutations in the reverse transcriptase and protease genes by comparing the gene sequences of a resistant virus to those of a wildtype strain that has previously been described. The efficacy of each ARV class and each individual ARV is threatened by specific mutations and resistance mechanisms. In retrospective studies of genotypic or phenotypic resistance testing, baseline resistance tests results were correlated with virological outcomes. There is some evidence from prospective studies that resistance testing may have some benefits when used to choose salvage regimens. However, problems in the areas of test interpretation, patient compliance, availabil-
\end{abstract}

\footnotetext{
${ }^{1}$ CHU Liège, Belgium

2 Service des Maladies Infectieuses et Tropicales, CHU Nice, France

${ }^{3}$ Laboratoire de Référence SIDA, CHU Liège, Belgium

${ }^{4}$ Service des Maladies Infectieuses, CHU Liège, Belgium

${ }^{5}$ Service des Maladies Infectieuses et Tropicales, CHU Nice, France
}

Address for correspondence :

P. Blaise,

Becco 618,

B-4910 Theux ity of active drugs, and technical test performance limit the usefulness of resistance testing in clinical practice.

This article reviews the mechanisms underlying HIV resistance, the principles of phenotypic and genotypic tests, and the use of these tests in clinical practice.

\section{INTRODUCTION}

In many HIV-infected patients, highly active antiretroviral therapy (HAART) consisting in combinations of potent antiretroviral drugs (ARVs) (1-4) can diminish the plasma viral load (HIV RNA concentration) to undetectable levels, thereby noticeably reducing HIV-related morbidity and mortality (5). Unfortunately, after one year of HAART, only 50 to $70 \%$ of patients enrolled in therapeutic trials still had undetectable viral loads (6). Virological failure, defined as resumption of measurable viral replication, is even more common in everyday clinical practice, particularly among patients who are experienced with ARV therapy (7). Many risk factors for virological failure are inherent in the virus itself, but others are related to the ARVs, the physicians, or the patient (Table I) $(8,9)$. The emergence of viral mutations associated with drug resistance is a pivotal phenomenon that is both a major cause of virological failure and the inescapable consequence of suboptimal viral control.

\section{EMERGENCE OF RESISTANCE MUTATIONS}

All developing organisms tend to escape from environmental pressures by selective replication of adapted strains rather than of susceptible strains. This natural 
TABLE I. Factors that contribute to failure of ARV therapy $(8,9)$ or disease progression

Virus-related factors

- development of resistance to ARVs

- transmission of virus resistant to ARVs

- emergence of syncytium-inducing virus

- genetic polymorphisms (?)

$\underline{\text { ARV-related factors }}$

- limited potency of ARVs

- poor penetration into sanctuaries (central nervous system, male genital tract, foetus...)

- drug-drug interactions

Physician-related factors

- choice of an inadequate ARV combination

- failure to perform or adequately use ARV resistance testing

- failure to assay ARVs

- failure to prevent side-effects

Patient-related factors

- poor adherence with therapy

- genetic heterogeneity of the co-receptors CCR5 and CXCR4

- efficacy of intracellular NRTI phosphorylation

- presence of cellular membrane-spanning proteins responsible for PI efflux

- ARV absorption/metabolism

- gradual immunological decline

(?) : Not fully demonstrated

selection process is a well-known cause of bacterial resistance to antimicrobial agents. Natural selection occurs at a particularly fast pace among HIV strains exposed to ARVs because of its high turnover associated with a high degree of genetic diversity (quasi-species) as in other lentiviruses. The propensity for mutations that underlies this genetic diversity is related to the high rate of reverse transcriptase errors, the absence of mechanisms for correcting these errors, and the rapid replication rates of HIV (10). This leads to the constitution of an extremely heterogeneous mixture of clones (quasispecies). The probability that one of these clones will be resistant to one or more ARVs is high. Mutations that confer ARV resistance, called primary mutations, usually slow the rate of viral replication of the affected strain (decreased fitness). Consequently, the prevalence of primary resistant viruses in a given patient before treat- ment is usually low: the variants with resistance mutations constitute small minorities within the quasi-species mixture. However, when treatment is started, the ARVs select these variants, whose speed of emergence depends both on the pre-treatment prevalence of the mutation and on the relative replicative advantage the mutation confers to the virus in the presence of the ARV (11). This measurable resumption of replication allows the virus to acquire not only other primary mutations but also secondary mutations that restore its replication potential, increasing the viral load (12). Primary and secondary mutations both occur in the genes encoding the reverse transcriptase (RT) and the protease (PR), which are the targets of currently available ARVs.

The risk of mutation emergence is correlated with the viral load under treatment (13). When the virus replicates more slowly (producing a low concentration of viral RNA), the risk that a resistance will emerge is smaller. On the contrary, a high viral load will be associated with an increased replication rate and a higher risk of emergence of resistance. Nevertheless, even with optimal treatment and a viral load below detectable levels, viral replication never stops completely. It follows that emergence of variants with resistance to ARVs is quite inevitable (14).

\section{ARV RESISTANCE TESTS}

The first report of HIV resistance to an ARV was a 1989 paper by Larder et al. describing phenotypically reduced susceptibility to zidovudine of HIV isolated during prolonged therapy (15). The genetic basis for this resistance was rapidly identified by comparisons of nucleotide sequences from resistant and susceptible viral strains (16). The mutations found within the RT gene showed some degree of reproducibility, allowing the development of rapid tests for detecting resistance to zidovudine, the only ARV available at the time.

"Genotypic resistance" is defined as the presence of mutations within the RT or P gene of viruses from patients experiencing virological failure or from laboratory HIV strains examined after subculturing with increasing drug concentrations. These mutations are identified by comparison with wild-type virus. Increase in the drug concentration that inhibits 50 percent (IC50) of growth of the tested HIV strain compared to that of a wild-type strain cultivated in parallel at varying drug concentrations defines the "phenotypic resistance". 


\section{Tests for genotypic resistance}

Testing for genotypic resistance involves examination of viral genome sequences amplified by the polymerase chain reaction (PCR). The virus is taken from the plasma of the patient. Mutations previously shown to confer resistance documented by phenotypic tests are looked for either by sequencing the entire RT and $\mathrm{P}$ genes or by examining specific codons for point mutations known to be associated with the emergence of resistance (17). Two sequencing kits (Visible Genetics,, and Applied Biosystems,) and one point mutation detection kit (Innogenetics,) are commercially available. However, many virology laboratories have developed their own home-brew sequencing methods. The main difficulty in genotypic resistance testing is the interpretation of the mutations. Different algorithms have been produced to automatically interpret the genetic sequence. For most drugs, a similar resistance interpretation was obtained when comparing 5 genotypic resistance interpretation algorithms. However, the algorithms largely diverge in the interpretation of resistance to stavudine, didanosine, and zalcitabine (18).

\section{Tests for phenotypic resistance}

Tests for phenotypic resistance consist in measuring the in vitro replication of the virus in the presence of varying concentrations of a given ARV. The ARV concentration needed to reduce replication by $50 \%\left(\mathrm{IC}_{50}\right)$ or $90 \%\left(\mathrm{IC}_{90}\right)$ is determined; thus, this method is analogous to determination of the minimal inhibitory concentration of an antimicrobial for a bacterial organism. Although the results can be expressed as absolute values (e.g., $\mathrm{IC}_{50}=800 \mathrm{nM}$ for the ARV X), they are usually reported as a comparison with the inhibitory concentration for a wild-type virus that serves as the reference (e.g., a strain with an $\mathrm{IC}_{50}$ of $800 \mathrm{nM}$ for $\mathrm{ARV} \mathrm{X}$ is reported as having eight-fold reduced susceptibility as compared to a reference virus whose $\mathrm{IC}_{50}$ to the same $\mathrm{ARV}$ is $100 \mathrm{nM}$ ).

In the past, phenotypic resistance was evaluated by culturing virus from the patient in peripheral blood mononuclear cells from healthy donors (19). This technique required 6 to 8 weeks, and the results varied with the ability of the blood cells to support growth of the HIV. These two problems have been overcome by the development of tests using recombinant HIV clones in which the RT and P genes are replaced by the corre- sponding genes of the HIV strain under study previously amplified by PCR on viral RNA harvested from the patient's plasma (20). Three phenotypic tests based on recombinant viruses are on the market (ViroLogic, , San Francisco; Virco,, Malines; and VIRalliance,, Paris). Phenotyping requires high laboratory skills and high security labs, which are not broadly available. Virco has recently released new cut-offs deemed to be more biologically relevant. Until now resistance tests have used somewhat arbitrary cut-offs that were, for example, based on the reproducibility of the tests themselves. The limitation of setting a single cut-off for all available drugs in this way is that it told the clinician very little about the significance of any change in susceptibility reported by a test, apart from it being large enough to be measured. In order to develop biologically relevant cut-off values for each drug, the $\mathrm{IC}_{50}$ values for 1,000 untreated patients as well as many thousands of samples of HIV-1 with no resistance mutations were measured (21). The average and the range of susceptibility were calculated for each drug. The cut-offs were then set at two standard deviations above the mean. This statistical term means that a test result falling above the cut-off can be said to be above the normal susceptible range with $97.5 \%$ confidence. Since the susceptibility of untreated and unmutated virus varied considerably from drug to drug, the new cut-offs are different for each drug. ViroLogic, San Francisco, California, has also defined new cutoffs based on clinical studies. Clinical cutoff values provide a direct correlation between resistance test results and clinical response. This means drug susceptibility standards based on actual clinical response to antiretroviral drugs. To determine clinical cutoffs, drug susceptibility is measured at treatment initiation. Treatment response, such as change in viral load, is monitored at predetermined study timepoints. Drug susceptibility is correlated with treatment response. Clinical cutoff value is determined by resistance levels associated with treatment failure (statistical analysis of overall trial results). Clinical cutoff values are currently being evaluated for all antiretroviral drugs, and are already available for Ziagen, Kaletra, d4T and ddl (22).

\section{Virtual Phenotype}

Another way to improve interpretation of genotypic informations is to confront them with phenotypic resistance data. Genotyping is more rapid and less expensive than phenotyping and, by detecting mutations in the 
genome, it can provide an early warning of potential resistance, even if full resistance to one or more drugs has not yet developed. The main limitation of genotyping is that it is not a direct measure of resistance. A genotypic test can reveal the mutations that have occurred in the relevant sequence of the HIV genetic code but then this information requires interpretation to predict which drugs the virus will and will not be sensitive to. With genotypic testing systems this is done by reference to lists of the main mutations and what effect they have on susceptibility to HIV drugs. Interpreting this information using tables or 'rules-based' algorithms, is difficult. Another approach combining genotypic information and phenotypic susceptibility has been developed. When a genotype for a patient sample is generated the genetic code for the RT-PR region is fed into a software system. This system identifies all the mutations that can affect resistance to each drug and then interrogates the database for genotypes from previous samples that match these patterns of mutations. When all the matches have been identified, the software retrieves the phenotypes for these samples and, for each drug, averages the data for all the matches. This produces a VirtualPhenotype ${ }^{\mathrm{TM}}$, with a fold change in $\mathrm{IC}_{50}$ for each drug. However, the number of samples matching the sequence can be limited for complex mutation patterns. The caveats of interpreting phenotypic resistance assays are also reproduced using this methodology. A study has shown the equivalence of virtual phenotype compared to phenotype in the choice of salvage therapy in highly ARV experienced patients (23).

\section{$\underline{\text { Advantages and disadvantages }}$}

Although they complement each other, genotypic and phenotypic tests each have their own advantages and disadvantages, which are listed in Table II. A study has shown that these tests suffer from lack of standardization: when specimens containing well-defined proportions of mutant viruses were sent to various reference laboratories, results in terms of sensitivity and specificity of the resistant mutant strains varied widely across laboratories (24). A new world-wide quality control has shown an improvement in the technical aspects of genotypic resistance testing but large divergence in the definition of resistance across laboratories (25).

Furthermore, both categories of tests involve conversion of RNA to DNA followed by PCR amplification. These procedures require a viral load greater than 1000 copies/ml. The detection threshold for HIV RNA
TABLE II. Advantages and disadvantages of genotypic and phenotypic resistance tests

\section{Phenotypic tests}

Advantages

- evaluate the net effect of mutations on susceptibility to ARV therapy

- provide information on cross-resistance

- give results as the $\mathrm{IC}_{50}$, which is more familiar to clinicians and, consequently, easier to interpret than genotypic test results

- provide information on resistance to a given ARV even when the genetic basis for that resistance has not yet been elucidated

\section{Disadvantages}

- insensitive to presence of minority mutated strains - costly

- so complex that availability is restricted to a few laboratories

- for most ARVs, cutpoints defining resistance or susceptibility have not yet been established or validated

- several weeks required to obtain results

\section{Genotypic tests}

\section{Advantages}

- fairly simple to use

- widely available

- results available rapidly

- can detect sentinel mutations not yet apparent phenotypically

\section{Disadvantages}

- not directly interpretable

- interpretation requires prior knowledge of the genetic determinants responsible for resistance

- interpretation requires an expert

- insensitive to the presence of minority mutated strains (point mutations assays are more sensitive than sequencing assays (63))

- cannot predict the effects of mutation-mutation interactions on the phenotype

is only 50 copies/ml. The HIV RNA concentration requiring a change in treatment in clinical practice remains to be defined but may be lower than 1000 copies/ml. 
The correlation between genotypic resistance and phenotypic resistance is poor for some nucleoside reverse-transcriptase inhibitors (NRTIs), fair for protease inhibitors (PIs), and excellent for non-nucleoside reverse-transcriptase inhibitors (NNRTIs) (26) . This correlation varies with the cutpoints chosen for phenotypic testing and with the algorithm used for genotypic testing.

\section{Nature of and resistance to the various ARVs}

The position and nature of the amino acid corresponding to the mutated codon play a key role in the development of resistance. The list of resistance mutations identified in vitro and/or in vivo is updated regularly (17), producing an increasingly detailed map of viral resistance. At the same time, our understanding of the mechanisms by which mutations cause resistance to ARVs has made huge strides. The three-dimensional structure of the enzymes RT and P has been defined. The structural enzyme changes generated by mutations and the effects of these changes on the affinity of the enzyme for its substrate, on its processivity, and on its mode of operation are gradually being elucidated.

$\underline{\text { Resistance to nucleoside reverse-transcriptase inhibitors }}$ (NRTIs)

\section{The mutations}

As an example, we will discuss mutations that confer resistance to zidovudine. Six substitutions, located in codons 41, 67, 70, 210, 215, and 219 of the RT gene, are known to confer resistance to zidovudine. These mutations emerge in a given order (27). Only in this order are the changes induced by the mutations consistent with the physico-chemical laws governing biological systems. Furthermore, this order of emergence is associated with the smallest possible effect on RT function.

The earliest mutation affects codon 70 and can emerge as early as 12 weeks after treatment initiation (28) . The viral population harbouring this mutation is rapidly replaced by more stable mutants, characterized by a change in codon 215 . Subsequently, the codon 70 mutation can reappear, and mutations can occur in codons 41, 67, and 219. In most patients, the codon 210 mutation occurs fairly late. Each mutation and combination of mutations confers its own decrease in susceptibility to zidovudine. Persistent viral replication in the presence of zidovudine results in gradual accumulation of mutations through a process of selection of the quasispecies characterized by greater resistance and higher replication potential, according to the Darwinian model (1). These mutations make the HIV increasingly resistant to zidovudine, so that viral replication persists and, eventually, the resistance extends to other ARVs (crossresistance). It has now been shown that some mutations confer resistance not only to zidovudine but also to d4T, abacavir, and probably ddI. Thus, mutations that confer resistance to thymidine analogs (thymidine analog mutations, TAMs) cause resistance to quite all NRTIs (29).

In some cases, a single mutation results in resistance to several or even all NRTIs. Two series of mutations conferring resistance to all available NRTIs have been identified. One is Q151M, which was first identified in patients taking zidovudine in combination with didanosine or zalcitabine (30) but since then has been found in patients on other NRTIs. Q151M is the first mutation to appear. It confers only small resistance on its own. Mutations at codons 77, 116, 62, and 75 rapidly develop which increase the resistance to zidovudine and to the other NRTIs (30-31). Resistance increases with the accumulation of the other mutations. Fewer data are available on the other mutation responsible for resistance to multiple NRTIs, T69S-SS. This mutation is characterized by insertion in codon 69 of six nucleotides, each of which encodes two serine residues. T69S-SS occurs as a result of prolonged treatment with several NRTIs, is less common than Q151M, and confers a high level of resistance to all currently available nucleoside and nucleotide analogues inhibitors of reverse transcriptase (32). This resistance mutation generally appears on the background of one or more members of the thymidine associated resistance mutations, particularly the T215Y (33).

Genotypic analysis of resistances remains complex. For instance, some mutations selected by one ARV result in resensitisation to another ARV to which the virus had become resistant. An example is M184V, which emerges during 3TC treatment but temporarily abolishes the zidovudine resistance-conferring effect of mutation T215Y (34).

\section{The mechanisms}

In parallel with the gradual accumulation of knowledge on the positions and types of resistance mutations, our understanding of the mechanisms underlying resistance is improving. For instance, the mechanism by which RT can become resistant to NRTIs has been elucidated. 
NRTIs, which are derivatives and analogues of naturally occurring nucleosides, induce competitive inhibition of RT (2). Once inside the cell, NRTIs are phosphorylated to triphosphates then compete with endogenous nucleosides for the RT catalytic site. When incorporated in the elongating DNA strand, NRTIs block further elongation, thereby halting viral replication. Thus, NRTIs are chain terminators. The mutations responsible for resistance to NRTIs act by modifying the three-dimensional conformation of RT, which then preferentially uses endogenous triphosphate deoxynucleosides: resistance of the enzyme is related to better discrimination between the natural substrate and the nucleoside analogue. This is one of the mechanisms underlying resistance of RT carrying the V75T mutation.

Another mechanism, called pyrophosphorolysis, has been described recently. This mechanism may explain the failure of some NRTIs to alter RT activity. As with all chemical reactions, construction of a DNA strand is a reversible process. In the presence of wild-type RT, nucleotide incorporation, which releases pyrophosphate, is strongly advantaged over nucleotide excision. However, some RT mutations make the enzyme capable of using an ATP molecule or a previously released pyrophosphate molecule to enhance the excision function. In this case, although excision remains disadvantaged as compared to incorporation, chain terminators are excised more frequently and, consequently, are less effective (35). Increase in excision of chain terminators is the main pathway for NRTIs resistance of a TAM bearing RT. On the contrary, resensitisation to zidovudine of RT bearing a TAM to which M184V is added (resistance to $3 \mathrm{TC}$ ) is related to a decrease in the pyrophosphorolysis capacity of the mutated RT, which can no longer excise the zidovudine incorporated at the end of the chain. This temporarily restores susceptibility to zidovudine. The third identified mechanism for $\mathrm{RT}$ resistance is an increase in processivity: all the functions of the enzyme, incorporation and excision, are accelerated.

Resistance to non-nucleoside reverse-transcriptase inhibitors (NNRTIs)

These ARVs also inhibit HIV replication by interfering with RT function, although they are structurally different from NRTIs. NNRTIs bind to the enzyme near its catalytic site, causing non-competitive inhibition of DNA strand polymerisation (36). In contrast to NRTIs, NNRTIs are active without prior intracellular metabolic changes.
Major resistances to NNRTIs can develop rapidly, sometimes within a few days of monotherapy initiation. Resistance is related to point mutations, usually in codons 98 to 108 or 181 to 190 of the RT. These mutations change the RT site that binds to NNRTIs decreasing its affinity for the compounds. K103N and Y181C are the most common mutations. Although NNRTIs vary widely in their chemical structure, cross-resistance is common. This is a factor contra-indicating sequential use of actual NNRTIs.

Interesting interactions between NRTIs and NNRTIs have been reported. The Y181C mutation, which confers resistance to NNRTIs, can increase viral susceptibility to zidovudine (37). Conversely, some viruses resistant to zidovudine and to abacavir show increased susceptibility to NNRTIs (38), via an as yet unidentified mechanism. Larger studies would be useful to determine the clinical relevance of these data.

\section{$\underline{\text { Resistance to protease inhibitors (PIs) }}$}

The HIV protease (PR) is a homodimer composed of two 99-residue chains. It cleaves transcribed viral polyproteins into six structural proteins (p17, p24, p2, p7, p1, and p6) and three enzymes (PR, RT, and integrase), thus allowing viral assembly (39). PIs compete with viral substrates for the active site of the PR. Resistance mutations to PIs emerge slowly and sequentially and are most common in patients with suboptimal plasma PI concentrations (40).

Although the $\mathrm{P}$ gene is small, more than 42 mutations conferring resistance to PIs have been identified (17). Some affect the substrate-binding site and cause the resistance itself. Others are secondary mutations that restore enzyme processivity, thus possibly enhancing the activity of the mutated $\mathrm{P}$ without directly affecting its binding to the inhibitor. Finally, other mutations, located outside the $\mathrm{P}$ gene, can compensate for the functional impairment in mutated $\mathrm{P}$ function. These last mutations affect different sites of the polyprotein, particularly the gag-pol cleavage site, where they restore sensitivity to cleavage by the defective mutated protease (41).

As a rule, susceptibility to PIs decreases gradually as mutations accumulate. Another effect of mutation accumulation is broadening of the resistance to other PIs, with cross-resistance or resistance to an entire class of ARVs. Some mutations, for instance L90M or the combination L10I + I84V, immediately confer a high level of resistance to most PIs, without requiring the accumulation of other mutations. 
Some RT gene mutations decrease RT activity, thereby weakening the replication potential of the mutated virus. This effect on replication seems even stronger with some $\mathrm{P}$ gene mutations and probably explains why some patients have fairly low viral loads despite a high level of resistance (8). These mutations modify enzymes that are crucial to HIV replication.

\section{$A R V$ resistance testing in clinical practice}

a) Retrospective studies indicating that $\mathrm{ARV}$ resistance testing is useful

In over a dozen retrospective studies, the information provided by genotypic or phenotypic testing at treatment initiation predicted virological outcomes (9). Furthermore, the Resistance Collaborative Group used a uniform framework to reappraise most retrospective and prospective studies in which data on HIV resistance at treatment initiation were available (42). The results showed that HIV resistance to the drugs used predicted virological success independently from other important clinical data such as prior treatment or viral load. b) Prospective studies using ARV resistance testing

Although there is a well-established association between resistance mutations and virological failure, the contribution of resistance testing to the management of patients experiencing virological failure is unclear. Several prospective studies have addressed this issue (Table IV) (43-50). Some found that resistance testing was beneficial in the short-term, although the advantage was fairly small (51). The viral load decrease in the group that underwent resistance testing was about $0.5-0.6 \log _{10}$ $/ \mathrm{ml}$, compared to the control group which is in the range of the decrease obtained with NRTI monotherapy on a wild-type strain. Similarly, the percentage of patients with undetectable viral load in the study group was only 15 to $20 \%$ higher than in the control group. The differences in these parameters were statistically significant in some studies but not in others $(51,52)$.

Numerous limitations affect these findings. In addition to the shortcomings of the resistance tests themselves, limitations present in all prospective studies included inadequacy of the interpretation algorithm available at the time, unavailability of drugs effective on resistant viruses, and lack of experience of the investiga-

TABLE III. Prospective studies in which ARV resistance testing was done in patients experiencing virological failure. From Demeter L (51)

\begin{tabular}{|c|c|c|c|c|}
\hline Study & $\begin{array}{l}\text { Design } \\
\text { copies/ml }\end{array}$ & $\begin{array}{l}\triangle \mathrm{VLLog}_{10} \\
\text { detection limit }\end{array}$ & $\begin{array}{l}\% \text { patients }< \\
\text { studied }\end{array}$ & Limiting factor \\
\hline Viradapt* $(43,53)$ & Geno/SOC & 1.04 vs. 0.46 & $29 \%$ vs. $14 \%$ & Pharmacology \\
\hline Vira $3001 * *(45)$ & Pheno/SOC & 1.72 vs. 2.21 & $45 \%$ vs. $34 \%$ & - \\
\hline GART* (46) & Geno + E/SOC & 1.19 vs. 0.61 & $34 \%$ vs. $22 \%$ & Advice from a virologist \\
\hline HAVANA* (39) & $\mathrm{SOC} \pm \mathrm{E} / \mathrm{Geno} \pm \mathrm{E}$ & 1.1 vs. 0.81 & $\begin{array}{l}58 \% \text { vs. } 42 \% \\
21 \% \text { vs. } 17 \%\end{array}$ & $\begin{array}{l}\text { Advice from } \\
\text { an expert }\end{array}$ \\
\hline ARGENTA (47) & $\mathrm{SOC}+\mathrm{E} / \mathrm{Geno}+\mathrm{E}$ & NA & $35 \%$ vs. $44 \%$ vs. $36 \% * * *$ & Compliance \\
\hline Narval* (49) & Geno/Pheno/SOC & 1.1 vs. 1.0 vs. 0.7 & $\mathrm{NA}^{* * *}$ & Interpretation algorithm \\
\hline Kaiser** (50) & Pheno/SOC & 0.4 vs. 0.2 & & - \\
\hline
\end{tabular}


tors or failure to obtain expert advice (52). In some of these studies, the reasons put forward to explain the small magnitude of testing-related benefits highlight several key aspects of patient management. For instance in the GART (genotypic ARV resistance testing) study (46), the patients whose physicians adapted the treatment according to the advice of virologists had better virological outcomes, as compared to the other patients. The benefits of obtaining expert advice were particularly conspicuous in the HAVANA trial (47), in which patients for whom expert advice was obtained were more likely to have an undetectable HIV RNA load, independently from whether genotypic testing was done. The ARGENTA trial (48) examined the effect of treatment compliance. Compliance of the patient with the treatment was a better predictor of successful viral load suppression than use of genotypic testing. The Viradapt trial (53) highlighted the importance of the pharmacological component as compared to resistance testing. Patients whose treatment was adapted based on genotypic test results and whose plasma PI concentrations were considered optimal were more likely to experience viral load suppression than the other patient subgroups. Finally, the Narval (49) trial pointed out the inadequacies of the genotype interpretation algorithm.

Furthermore, the ARGENTA (48), Narval (49), and Kaiser (50) studies showed that resistance testing was less useful in patients with a history of multiple virological failures. This is probably because no ARVs effective on multiresistant HIV strains are available.

\section{c) Limitations to the use of ARV resistance testing}

Resistance tests themselves and their modalities of use are still at the early stages of development. Quality control studies have shown that resistance testing has

\section{TABLE IV. Limitations of genotypic tests related to the sequencing tool and to interpretation of results}

Limitations related to the tool

- PCR amplification possible only if viral load $>1000$ copies $/ \mathrm{ml}$

- less reliable if presence of non-B viral subtypes

- poor sensitivity for detecting minority strains contributing 20 to $50 \%$ of the overall mixture ( $>>)$

- the resistance analysis method has not been validated

- the assistance of an expert is needed to perform the test and to read the sequence

- costly

- no information available about the new ARV classes that will become available in the very near future (e.g., fusion inhibitors)

- testing explores only some HIV genes, providing no information on other regions ( $g a g$-pol) that are involved in resistance

- mainly identifies drugs likely to be ineffective

- major discrepancies between phenotypic and genotypic results on susceptibility, especially for nucleoside analogues

- does not take into account mutation-mutation interactions, which can result in resensitisation or in appearance of another biological antiviral mechanism (effect on fitness, or pyrophosphorolysis for instance of mutation M184V)

- limited value in the event of planned or unplanned treatment discontinuation because misses resistances present in minority strains or stored in proviral DNA incorporated in the cell genome

Limitations related to use of the genetic sequence result in clinical practice

- need for the advice of an expert in the clinical and treatment setting

- lack of clinical-virological correlation between presence of a given mutation and virological outcome under a given drug (interpretation algorithms based on in vitro data and on clinical studies of monotherapy, whereas in practice patients take drug combinations): inadequacy of interpretation algorithms

- lack of drugs effective on resistant viruses

- time lag between access to new drugs and knowledge of mutations conferring resistance to these drugs 
improved over time $(24,25,54)$. Table IV lists remaining shortcomings of the tests and their modalities of use.

Interpretation, the Achilles tendon of resistance testing, is changing constantly. TAMs have been shown to cause resistance not only to zidovudine but also to d4T (55) and even to ddI (56). It has been suggested that overuse of $\mathrm{d} 4 \mathrm{~T}$ and $\mathrm{ddI}$ in the genotypic testing group of the Narval study was beneficial in that it reduced the use of new ARVs; however, this may be a limitation to the study, as the resistance test results were not used optimally, according to current knowledge. The new genotypic test interpretation algorithm developed by the ANRS (Agence Nationale de Recherche sur le SIDA, France) takes TAMs into account by indicating as the result "resistance to zidovudine, d4T, and also ddI". Data from the analysis of virological outcomes in the control arm of the Narval trial have been used to fine-tune the interpretation of genotypic resistance to abacavir and amprenavir (56).

\section{d) Current recommendations regarding use of resistance testing}

The first consensus report about the indications for ARV resistance testing was published in 1998 (57). The increased availability of resistance tests and evidence that these tests have some efficacy in clinical practice have prompted the development of new recommendations in Europe (58-61) and in the United States (62).

The prevalence of the various HIV-1 subtypes, transmission of resistant strains, availability of tests, reimbursement of tests by health care insurance, and many other factors vary from one country to the next. Consequently, each country has developed its own recommendations. Table V shows the recommendations for Belgium.

Testing is not recommended in chronically HIV-infected patients prior to treatment initiation because the resistant virus population possibly found in this situation is so small that it escapes isolation. Similarly, in patients experiencing virological failure, resistance testing should be done before the treatment is stopped or changed, as afterwards the prevalence of the resistant variants rapidly drops to undetectable levels.

\section{CONCLUSION}

The management of patients infected with the HIV has made huge strides forward in the last few years, particularly with the advent of new ARVs. The emergence of viral variants that are resistant to ARVs is related not only to the high replication and mutation rates of the HIV but also to poor compliance with therapy and to use of insufficiently suppressive drug combinations. Emergence of resistances remains the main obstacle to long-lasting treatment success. Identifications of viral mutations that confer specific resistance to a given ARV should allow clinicians to choose the drug combinations that maximally suppress viral replication. Retrospective studies have shown that phenotypic or genotypic viral resistance at treatment initiation predicted the virological response to the treatment used.

\section{TABLE V. Belgian recommendations for ARV resist- ance testing (61)}

ARV resistance testing should be performed in situations such as those listed below:

1. Virological treatment failure, when a change in treatment is being considered; the usefulness of the resistance test will depend on remaining treatment options. If the virus shows multidrug resistance, the test will have little impact on treatment decisions.

2. Treatment initiation in a child. Resistant strains can be transmitted vertically.

3. Treatment initiation during pregnancy. Optimal treatment is indispensable to minimize the risk of vertical transmission.

ARV resistance testing should be considered in situations such as those listed below:

1. Treatment initiation in a treatment-naive patient. Priority should be given to treatment-naive patients undergoing seroconversion, contamination having apparently occurred in the recent past. Detection of resistant mutants may be impossible later during the course because of the decreased replication potential of mutants. If testing is not done at this time, plasma should be stored whenever possible.

2. Post-exposure prophylaxis. Every effort should be made to obtain a sample from the HIV-infected contact. Treatment cannot be postponed until the results of ARV resistance testing are available but, if needed, can be adapted based on the results. 
However, prospective studies of genotypic testing have produced mixed results. Numerous limitations related to the tests themselves and to the way they are used have been recognized. Improvements in resistance testing, in our knowledge of the mechanisms underlying resistance, and in resistance test interpretation can be expected to optimize ARV therapy in the near future. However, therapeutic failure is multifactorial. Therefore, multidisciplinary management of HIV infection should be the reference standard and the most useful recommendation at present.

\section{REFERENCES}

1. Moutschen M, Nkoghe D, Leonard P, Demonty J. Comment je traite une infection par le HIV. I. Bases pathogéniques des choix thérapeutiques. Rev Med Liège. 1997;52:622-4

2. Moutschen M, Nkoghe D, Leonard P, Demonty J. Comment je traite une infection par le HIV. II. Inhibiteurs nucléosidiques de la transcriptase reverse. Rev Med Liège. 1997;52:750-2

3. Nkoghe D, Moutschen M, Leonard P, Demonty J. Comment je traite une infection par le HIV. III. Les inhibiteurs non nucléosidiques de la transcriptase reverse. Rev Med Liège. 1999;54:909-11

4. Nkoghe D, Moutschen M, Demonty J et le Groupe de Travail de Liège sur l'Infection par le VIH. Recommandations pour la prise en charge du patient infecté par le HIV. I. L'adolescent et l'adulte. Rev Med Liège. 2000;55:417-23

5. Palella FJ, Delaney KM, Moorman AC et al. Declining morbidity and mortality among patients with advanced human immunodeficiency virus infection. $N$ Engl J Med. 1998;338:853-60

6. Staszewski S, Morales-Ramirez J, Tashima KT et al. Efavirenz plus zidovudine and lamivudine, efavirenz plus indinavir, and indinavir plus zidovudine and lamivudine in the treatment of HIV-1 infection in adults. Study 006 Team. $N$ Engl J Med. 1999;341:1865-73

7. Deeks SG, Hecht FM, Swanson M et al. HIV RNA and CD4 cell count reponse to protease inhibitor therapy in a urban AIDS clinic: reponse to both initial and salvage therapy. AIDS. 1999;13:F3543

8. Kuritzkes DR. Clinical implications of antiretroviral resistance. Disponible sur le site "http://HIV.medscape.com/Medscape/HIV/ ClinicalMgmt/CM.v13/pnt-CM.v13.htlm“ en février 2001

9. Clevenbergh P, Durant J, Garraffo R, Chaillou S, Cua E, Dellamonica P. Les multiples aspects de la résistance du HIV aux antirétroviraux. Méd Mal Infect. 2000;30:551-64

10. Coffin JM. Genetic variation in AIDS viruses. Cell. 1986; 46 :14

11. Coffin JM. HIV population dynamics in vivo: Implications for genetic variation, pathogenesis and therapy. Science. 1995;267:483-9

12. Kellam P, Boucher CAB, Tijnagel JMGH, Larder BA. Zidovudine treatment results in the selection of human immunodeficiency virus type 1 variants whose genotypes confer increasing levels of drug resistance. J Gen Virol. 1994;75:341-51

13. Drusano GL, Bilello JA, Stein DS et al. Factors influencing the emergence of resistance to indinavir: role of virologic, immuno- logic, and pharmacologic variables. J Infect Dis. 1998;178:3607

14. Rubio A, Gomez-Cano M, Puig T et al. Presence of genotypic resistance in nucleoside analogue-treated HIV-1-infected patients with undetectable viral load. Antivir Ther. 1999;4:45-9

15. Larder BA, Darby G, Richman DD. HIV with reduced sensitivity to zidovudine (AZT) isolated during prolonged therapy. Science. 1989;243:1731-34

16. Larder BA, Kemp SD. Multiple mutations in HIV-1 reverse transcriptase confer high-level resistance to zidovudine (AZT). Science. 1989;246:1155-8

17. Loveday C. Resistance testing in HIV disease management. IAPAC Symposium, October 10, 1999, Vienna, Austria. Available: http://www.iapac.org/conferences/vienna99

18. Japour AJ, Mayers DL, Johnson VA et al. A standardized peripheral blood mononuclear cell culture assay for the determination of drug susceptibilities of clinical human immunodeficiency virus type-1 (HIV-1) isolates. Antimicrob Agents Chemother. 1993;37;1095-101

19. Kellam P, Larder BA. Recombinant virus assay: a rapid phenotypic assay for assessment of drug susceptibility of human immunodeficiency virus type 1 isolates. Antimicrob Agents Chemother. 1994;38:23-30

20. Schuurman R, Demeter L, Reichelderfer P, Tijnagel J, de Groot $\mathrm{T}$, Boucher C. Worldwide evaluation of DNA sequencing approches for identification of drug resistance mutations in the human immunodeficiency virus type 1 reverse transcriptase. $J$ Clin Microbiol. 1999;37:2291-6

21. Schinazi RF, Larder BA, Mellors JW. Mutations in retroviral genes associated with drug resistance. Int Antiviral News. 1997;5:129-42

22. Richman DD. The implications of drug resistance for strategies of combination antiretroviral chemotherapy. Antiviral Res. 1996;29:31-3

23. Shirasaka T, Kavlick MF, Ueno T et al. Emergence of human immunodeficiency virus type 1 variants with resistance to multiple dideoxynucleosides in patients receiving therapy with dideoxynucleosides. Proc Natl Acad Sci USA. 1995;92:2398402

24. Iversen AKN, Shafer RW, Wehrly K et al. Multidrug-resistant human immunodeficiency virus type 1 strains resulting from combination antiretroviral therapy. J Virol. 1996;1086-90

25. de Jong JJ, Goudsmit J, Lukashov VV et al. Insertion of two amino acids combined with changes in reverse transcriptase containing tyrosine-215 of HIV-1 resistant to multiple nucleoside analogs. AIDS. 1999; 13:75-80

26. St Clair MH, Martin JL, Tudor-Williams G et al. Resistance to ddI and sensitivity to AZT induced by a mutation in HIV-1 reverse transcriptase. Science. $1991 ; 253: 1557-9$

27. Arion D, Kaushik N, Mc Cormick S et al. Phenotypic mechanism of HIV-1 resistance to 3'-azido-3'-deoxythymidine (zidovudine) [Abstract 14]. Antiviral Ther. 1998;3(Suppl1):13

28. Spence RA, Kati WM, Anderson KS, Johnson KA. Mechanism of inhibition of HIV-1 reverse transcriptase by nonnucleoside inhibitors. Science. 1995;267:988-93

29. Larder BA. 3'-azido-3'-deoxythymidine resistance suppressed by a mutation conferring human immunodeficiency virus type 1 resistance to nonnucleoside reverse transcriptase inhibitors. Antimicrob Agents Chemother. 1992;36:2664-9

30. Whitcomb J, Deeks S, Huang W et al. Reduced susceptibility to NRTI is associated with hypersensibility to NNRTI in virus from 
HIV-1-infected patients. Program and abstracts of the $7^{\text {th }}$ Conference on Retroviruses and Opportunistic Infections; January 30 - February 3, 2000 San Fransisco. California. Abstract 243

31. Swanstrom R, Willis J. Retroviral gene expression: synthesis, processing and assembly of viral proteins. In: Varmus HE, Coffin J, Hughes S. Retroviruses. Cold Spring Harbor Laboratory Press. 1997; 7:263-334

32. Condra JH, Schleif WA, Blahy OM et al. In vivo emergence of HIV-1 variants resistant to multiple protease inhibitors. Nature. 1995;374:569-71

33. Zhang YM, Imamichi H, Imamichi T et al. Drug resistance during indinavir therapy is caused by mutation in the protease gene and in its gag substrate cleavage sites. J Virol. 1997;71:6662-70

34. DeGruttola V, Dix L, D'Aquila R, et al. The relation between baseline HIV drug resistance and response to antiretroviral therapy: re-analysis of retrospective and prospective studies using a standardized data analysis plan. Antiviral Ther. 2000;5:4148

35. Durant J, Clevenbergh P, Halfon P et al. Drug-resistance genotyping in HIV-1 therapy: The VIRADAPT randomised controlled trial. Lancet. 1999;353:2195-9

36. Clevenbergh P, Durant J, Halfon P et al. Persisting long-term benefit of antiretroviral genotypic guided treatment for HIV-infected patients failing HAART. Antiviral Ther. 2000;5:65-70

37. Cohen C, Kessler H, Hunt S, et al. Phenotypic resistance testing significantly improves response to therapy: Final analysis of a randomized trial (VIRA3001). Antiviral Ther. 2000;5(Suppl3):67

38. Baxter JD, mayers DL, Wentworth DN et al. Final results of CPCRA 046: a pilot study of antiretroviral management based on plasma genotypic antiretroviral resistance testing (GART) in patients failing antiretroviral therapy. Antiviral Ther. 1999;4(suppl1):43. Abstract 61

39. Tural C, Ruiz L, Holtzer C, et al. The potential role of resistance decision support software with or without expert advice in a trial of HIV genotyping versus standard of care-the Havana Trial. Program and abstracts of the 40th Interscience Conference on Antimicrobial Agents and Chemotherapy; Toronto, Ontario, Canada; September 17-20, 2000. Abstract L-10

40. De Luca A, Antinori A, Cingolani A, et al. A prospective, randomized study on the usefulness of genotypic resistance testing and the assessment of patient-reported adherence in unselected patients failing potent HIV therapy (ARGENTA): final 6-month results. 8th Conference on Retroviruses and Opportunistic Infections; February 4-8, 2001; Chicago, Illinois. Abstract 433

41. Meynard JL, Vray M, Morand-Joubert L, et al. Impact of treatment guided by phenotypic or genotypic resistance tests on the response to antiretroviral therapy: a randomized trial (NARVAL, ANRS 088). Antiviral Ther. 2000;5(suppl 3):67-68

42. Melnick D, Rosenthal J, Cameron M, et al. Impact of phenotypic antiretroviral drug resistance testing on the response to salvage antiretroviral therapy (ART) in heavily experienced patients. 7th Conference on Retroviruses and Opportunistic Infections. January 30-February 2, 2000; San Francisco, California. Abstract 786
43. Demeter L. Drug resistance and the management of treatment failure. Program and abstracts of the 7th Conference on Retroviruses and Opportunistic Infections; January 30-February 2, 2000; San Francisco, California. Abstract S32

44. Clevenbergh P, Blaise P, M Okome N'Koumou, Dellamonica P. Les balbutiements de l'analyse génotypique de la résistance du VIH aux antirétroviraux : la difficile application des performances de la biologie moléculaire à l'efficacité thérapeutique. Med Ther 2002; 8:36-41

45. Durant J, Clevenbergh P, Garraffo R et al. Importance of protease inhibitor plasma levels in HIV-infected patients treated with genotypic-guided therapy: pharmacological data from the Viradapt Study. AIDS. 2000;14:1333-9

46. Schuurman R, Brambilla DJ, De Groot D, et al. Second worldwide evaluation of HIV-1 drug resistance genotyping quality, using the ENVA-2 panel. Program and abstracts of the 39th Interscience Conference on Antimicrobial Agents and Chemotherapy; September 26-29, 1999; San Francisco, California. Abstract 1168

47. Calvez V. Zidovudine associated resistance mutations can be selected in patients receiving long-term exposure to stavudine. Antiviral Therapy. 1999; 4 (Supp.1), abstract 38

48. Costagliola D, Descamps D, Calvez V. Presence of thymidineassociated mutations and response to $\mathrm{d} 4 \mathrm{~T}$, abacavir and ddI in the control arm of the NARVAL ANRS 088 trial. 8th Conference on Retroviruses and Opportunistic Infections; February 48, 2001; Chicago, Illinois. Abstract 450

49. Hirsch MS, Conway B, D'Aquila RT et al. Antiretroviral drug resistance testing in adults with HIV infection. JAMA. 1998;279:1984-91

50. Soriano V, Ledesma E and the Spanish Drug Resistance Panel. Second spanish consensus on the use of drug resistance testing in clinical practice. AIDS. 2000;2:111-8

51. Prise en charge thérapeutique des personnes infectées par le HIV. Rapport d'experts 1999 sous la direction du Professeur JF Delfraissy. Paris. Flammarion Médecine-Sciences;1999:35-50

52. BHIVA Writing Committee on behalf of the BHIVA Executive Committee. British HIV Association (BHIVA) guidelines for the treatment of HIV-infected adults with antiretroviral therapy. HIV Med. 2000;1:76-101

53. Consensus belge de thérapeutique et de surveillance du patient adulte infecté par le VIH, Réunion du $1^{\text {er }}$ décembre 2000 présidée par N. Clumeck (Service des Maladies Infectieuses, CHU St Pierre Bruxelles) et R. Colebundus (Département de Médecine Interne, ITG Anvers)

54. Hirsch MS, Brun-Vézinet F, D’Aquila RT et al. Antiretroviral drug resistance testing in adult HIV-1 infection. Recommendations of an International AIDS Society-USA Panel. JAMA. 2000;283:2417-26 